\title{
COMPARISON OF VERTICAL HANDOVER MECHANISMS USING GENERIC QOS TRIGGER FOR NEXT GENERATION NETWORK
}

\author{
Rajender Kumar ${ }^{1}$ and Brahmjit Singh ${ }^{2}$ \\ ${ }^{1}$ ECE Department, NIT Kurukshetra, INDIA, IEEE Member \\ rkumaranitkkr.ac.in \\ ${ }^{2}$ ECE Department, NIT Kurukshetra, INDIA, IETE Member \\ brahmjitanitkkr.ac.in
}

\begin{abstract}
Next Generation Network (NGN) is envisage to integrate heterogeneous wireless systems. NGN is expected to have Quality as the major challenging issue. More specifically, NGN aims at providing guaranteed Quality of Service $(Q o S)$ and Quality of Experience $(Q o E)$ i.e. what quality a network provider can offer (or claims) \& what quality user experiences. NGN user may be interested in changing its network operator or is forced to change the serving access point (AP) or base station (BS) in order to achieve a given level of QoE. Seamless transfer of user's service from existing operator to a new operator bearing dissimilar radio access technology is called Vertical Handover (VHO). VHO decision may depend on the popular signal to noise ratio (SNR) criteria or other performance metrics like bit error rate, delay, jitter etc or combination of these metrics. Performance of candidate target network depends on the type of traffic i.e. whether conversational, streaming, interactive or background is implemented. Therefore to optimise VHO mechanism, all these metrics \& traffic types should be evaluated. Paper elucidates contemporary VHO approaches i.e. hybrid ANP/ RTOPSIS \& Oliver blume method. Different Interworking scenarios \& NGN testbeds are prepared considering Wi-Fi, WiMAX, UMTS etc. as the candidate networks. Neuro-fuzzy based MCDM weight assignment technique is applied \& overall network cost function is computed based on Qualnet simulation. At the end of the paper investigation suggests that Blume algorithm is better than the hybrid ANP mechanism because it is simple to implement, more efficient (40\% more accurate), quickly computed (20 times less) and more dynamic (application oriented).
\end{abstract}

\section{KEYWORDS}

Comparison, MCDM, QoS metric, NGN testbeds, VHO mechanism

\section{INTRODUCTION}

Next Generation Network (NGN) will inexorably integrate triple-play services, which means that all traffic classes of voice, video and data will be managed to meet the particular Quality of Service (QoS) requirements, such as strict packet delay, jitter and loss guarantees.[1] It is well known that NGN will inevitably include Internet as the major backbone network i.e. fourth generation $(4 \mathrm{G})$ is proposed to be fully IP centric. It is believed that the deployment of NGN and the provisioning of triple-play services will eventually not only benefit the Internet users with richer contents, but also increase ISP (Internet Service Provider) revenues by acquiring much higher per-subscriber profit. The Internet has been evolving in recent years to adapt with the emerging abundant applications [2].

\subsection{VHO Necessity :}

Next Generation Network user may be interested in changing its network operator or is forced to change the serving access point (AP) or base station (BS) in order to achieve a given level of Quality of Experience (QoE) . Seamless transfer of user's service from existing operator to a new operator bearing dissimilar radio access technology is called Vertical Handover (VHO). Vertical 
Handover (VHO) [3] is a mechanism in which user maintains connection when switched from one Radio Access Network (RAN) technology to another RAN technology (e.g., from WLAN/H2 to UMTS and vice versa (see fig.1). [4][5]. VHO is different from conventional horizontal handover where the mobile devices move from one base station to another within the same network (RAN). In VHO, a session is seamlessly handed over to a new RAN in an interoperable region based on a criterion dictating the signal quality. This is called 'triggering' the VHO initiation phase. Wireless channel estimation is associated with PHY and MAC layer therefore it is called L1/L2 triggering, [6]. We may include metrics/ triggers of other layers for VHO execution and can be view VHO as cross layer design problem.

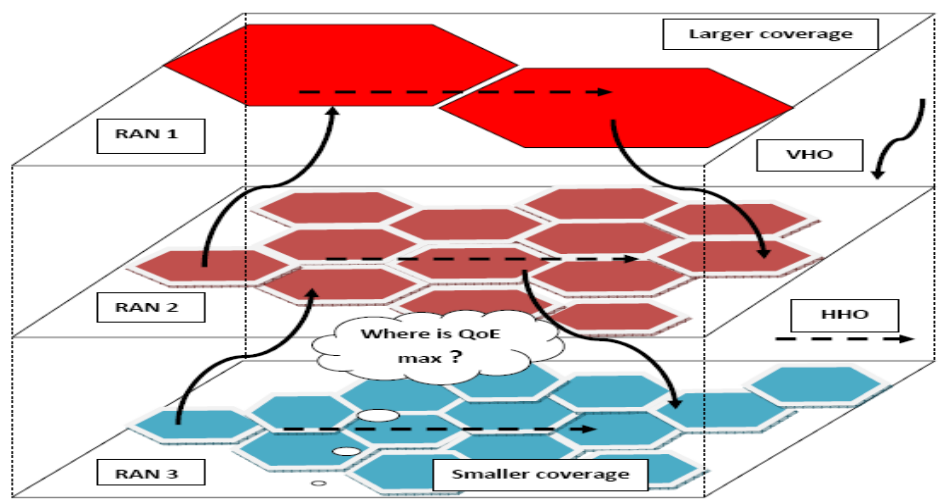

Figure-1 Vertical Handover in NGN

Table- 1

\begin{tabular}{|c|c|c|c|c|}
\hline $\begin{array}{c}\text { Wireless } \\
\text { Networks }\end{array}$ & $\begin{array}{c}\text { Wireless } \\
\text { Standards }\end{array}$ & Data-Rate & $\begin{array}{c}\text { Frequency } \\
\text { Band }\end{array}$ & Modulation Scheme \\
\hline Wi-Fi & $802.11 \mathrm{~b}$ & $11 \mathrm{Mbps}$ & $2.4 \mathrm{GHz}$ & $\begin{array}{c}\text { OFDM }>20 \mathrm{Mbps}, \\
\text { DSSS with CCK < 20 Mbps }\end{array}$ \\
\hline WiMax & $802.16 \mathrm{a}$ & $\begin{array}{c}75 \mathrm{Mbps}(\mathrm{UL}) \\
25 \mathrm{Mbps}(\mathrm{DL})\end{array}$ & $2-11 \mathrm{GHz}$ & OFDM \\
\hline MANET & - & $2-54 \mathrm{Mbps}$ & & \\
\hline Satellite & - & $\begin{array}{c}<\mathrm{Gbps} \\
1 \mathrm{Mbps}(\mathrm{avg})\end{array}$ & $\begin{array}{c}12 \mathrm{GHz}(\mathrm{UL}) \\
14 \mathrm{GHz}(\mathrm{DL})\end{array}$ & BPSK,QPSK,16 QAM \\
\hline UMTS & - & $2 \mathrm{Mbps}$ & $1.95 \mathrm{GHz}(\mathrm{UL})$ & QPASK \\
& - & $2.15 \mathrm{GHz}(\mathrm{DL})$ & QPSK \\
\hline UMTS+HSDPA & - & $\begin{array}{l}1.95 \mathrm{GHz}(\mathrm{UL}) \\
2.15 \mathrm{GHz}(\mathrm{DL})\end{array}$ & PAM \\
\hline Wired LAN & 802.3 & $100 \mathrm{Mbps}$ & $2.4 \mathrm{GHz}$ & \\
\hline
\end{tabular}

\subsection{NGN candidate networks}

In the evolution of NGN standards, there were four major factors that classified wireless mobile networks i.e. data rate, mobility, bandwidth \& coverage. Data rate was dependent on bandwidth and mobility was associated with coverage. Thus, based on data rate \& coverage networking standards/ technologies were divided i.e. standards having lower data rate/ larger coverage and standards of higher data rate but smaller coverage.[table-1] Due to the extensive research, few standards are coming up claiming higher data rates \& larger coverage area like WiMAX \& LTE 
proposed by IEEE $802.16 \& 3 \mathrm{GPP}-2$ resp. [7][8]. In paper, Wi-Fi, Wi-Max, UMTS, UMTS+HSDPA, Satellite, MANET and wired LAN are considered for investigation.

$>$ Wi-Fi : Wi-Fi is abbreviation of Wireless Fidelity is a set standard in the wireless domain based on IEEE 802.11 specifications. In simple terms it allows a user to connect to the local area network and also have access to the internet without any wires connected to the computer. It is faster than a typical cable modem connection hence giving all the flexibility that the user needs in terms of connectivity and bandwidth [5].

D WiMAX: WIMAX is Worldwide Interoperability for Microwave Access. WIMAX is an emerging technology fulfilling QoS requirements of the customers. WiMAX signals have the property to adopt the atmospheric conditions everywhere. WIMAX electromagnetic waves also offer the support of adoptive coding and different operation modes, so voice and data services can easily be transported by WIMAX network platform [7].

DSPDA: High Speed Downlink Packet Access is introduced in (3G) wireless network obtain high speed data rates. HSDPA is a modified interface version of UMTS in 3GPP. It provides not only down link packet access but also it can be used for uplink data up to 14 Mbps per user.

UMTS: Universal Mobile Telecommunication System is a third generation (3G) mobile communication system that provides a range of broadband wireless and mobile communication services. UMTS's target is to build an all-IP network by extending the $2 \mathrm{G}$ GSM/GPRS system and using complex technologies including Code Division Multiple Access (CDMA), Asynchronous Transfer Mode (ATM), and Internet Protocol (IP) [8].

MANET: Mobile Ad-hoc Network (MANET) consists of mobile hosts equipped with wireless communication devices. Due to the mobility of wireless hosts, each host needs to be equipped with the capability of an autonomous system, or a routing function without any statically established infrastructure or centralized administration. The mobility and autonomy introduces a dynamic topology of the networks not only because end-hosts are transient but also because intermediate hosts on a communication path are transient [9].

$>$ LAN: Wired LAN or IEEE 802.3 is a collection of IEEE standards defining the Physical Layer and Data Link Layer's media access control (MAC) sublayer of wired Ethernet. Physical connections are made between nodes and/or infrastructure devices (hubs, switches, routers) by various types of copper / fiber cable.

> SAN : SAN is Satellite Area Network. Satellite is a communication device used for high scale broadcast and monitoring purpose that may be stationary or revolving in an orbit. Modern satellite systems use advanced technology to provide broadband data service to areas unserved or underserved by other telecommunications systems. Satellite systems provide rapid setup/teardown of end-systems in field-deployable systems, and have an easily-satisfied requirement for high-elevation line-of-sight communication.

\subsection{Multimedia traffic}

Multimedia traffic can be classified into four categories according to the class of services (TS23.107) defined by 3GPP [10]-[11] 
Conversational Traffic: The typical applications of this class are VoIP and video conferencing. Real-time conversation is always performed between peers (or groups) of live (human) end-users. This is the only traffic where the required QoS characteristics are strictly given by human perception. e.g. VOIP application.

$>$ Streaming Traffic: When the user is looking at (listening to) real-time video (audio), the scheme of real-time streams applies. The real-time data flow is always aiming at a live (human) destination. It is a one-way transport. e.g. CBR application.

$>$ Interactive Traffic: When the end-user, that is either a machine or a human, is online requesting data from remote equipment (e.g. a server), this type of traffic applies. Examples of human interaction with the remote equipment are: Web browsing, database retrieval, server access. Examples of machines interaction with remote equipment are: polling for measurement records and automatic database enquiries (tele-machines). e.g. MCBR application.

$>$ Background Traffic: When the end-user, that typically is a computer, sends and receives data-files in the background, this type of data transmission is called background traffic. Examples are background delivery of e-mails, SMS, download of databases and reception of measurement records. e.g. FTP application

Section 1 of the paper introduces the NGN candidate networks, VHO necessity and different types of application based traffic classes. Section 2 is a literature survey covering contemporary VHO approaches i.e. hybrid ANP/ RTOPSIS \& Oliver blume method followed by generic QoS triggers. Section 3 provides neuro-fuzzy based MCDM weight assignment technique \& computes an overall cost function based on Qualnet simulation. Section 4 simulates the Interworking scenario \& NGN testbeds. Section 5 presents performance analysis of three mechanisms based on the network cost and elucidates comparison of VHO mechanisms.

\section{LITERATURE SURVEY}

\subsection{VHO mechanism evolution}

2005,Q. Y. Song et al. [12] proposed a network selection scheme for the integration of UMTS and WLAN. Analytic hierarchy process (AHP) is applied to decide the relative weights of evaluative criteria set according to user preferences and service applications, and grey relational analysis (GRA) is adopted to rank the network alternatives.

$>2006$,Olga Ormond [13] proposed a utility-based algorithm that accounts for user time constraints, estimates complete file delivery time (for each available network) and selects the most promising access network based on consumer surplus (CS) difference.

$>$ 2007,Feng and Furong (China) [14] presented the quality of network $\mathrm{i}$ at a certain time considered as a function of the available bandwidth it can offer ( $\mathrm{Bi}$ ), the timeliness value (Ti), and error rate value( $\mathrm{Ei})$. $\quad \mathrm{Q}_{\mathrm{i}}=\mathrm{f}\left(\mathrm{B}_{\mathrm{i}}, \mathrm{T}_{\mathrm{i}}, \mathrm{E}_{\mathrm{i}}\right)$

$>$ 2008, Wei Shen and Zeng, the cost to use network $N_{i}$ for an originating call is defined as

$$
\mathrm{C}_{\mathrm{i}}=\mathrm{wg} \cdot \mathrm{G}_{\mathrm{i}}+\mathrm{ws} \cdot \mathrm{S}_{\mathrm{i}} \text {, for } \mathrm{i}=1,2, \ldots, \mathrm{M}(1)
$$

where $\mathrm{G}_{\mathrm{i}}$ is the complementary of the normalized utilization of network $\mathrm{Ni}, \mathrm{Si}$ is the relative received signal strength from network $\mathrm{N}_{\mathrm{i}}$, and wg $(0 \leq \mathrm{wg} \leq 1)$ and ws $(0 \leq \mathrm{ws} \leq 1)$ are the weights that provide preferences to $\mathrm{G}_{\mathrm{i}}$ and $\mathrm{S}_{\mathrm{i}}$, respectively [15].

$>$ 2009, Oliver Blume [16] filed a patent on 'Methods of Identifying a radio link'.

As Wei proposed cost function based on only signal strength and network utilization and didn't included user's different traffic type. Feng proposed the decision based on bandwidth, 
timeliness and error rate but not included delay profile. Therefore algorithm has scope of improvement. Olga Ormond proposed consumer surplus based algorithm emphasising on cost rather than quality. Application / traffic class is an integral part of VHO Decision therefore it is included in analysis of VHO decision \& accuracy.

$>$ 2010, Rajender Kumar et al. [6] proposed 'EVM' as a new QoS trigger to initiate VHO for OFDM based wireless networks. Choi et al. [17] carried performance evaluation of opportunistic VHO considering on-off characteristics of VoIP Traffic. [18]

\subsection{Generic QoS triggers}

In wireless communication there are a number of parameters on the basis of which we can determine and decide the QoS. Some of them are bit error rate (BER), jitter, latency, error vector measurement $(\mathrm{EVM})$, throughput ,delay etc. The paper considers and analyses throughput, endto-end delay, Jitter, BER and SNR

\section{Throughput:}

Throughput or network throughput is the average rate of successful message delivery over a communication channel. These data may be delivered over a physical or logical link, or pass through a certain network node. Throughput is usually measured in bits per second (bit/s or bps), and sometimes in data packets per second or data packets per time slot.

Throughput $=[($ Total Bytes Sent * 8) $/($ Time Last Packet Sent - Time First Packet Sent $)]$ where 'time' is in seconds.

\section{$>$ End-to-End Delay:}

End-to-end delay indicates the length of time taken for a packet to travel from the CBR (Constant Bit Rate) source to the destination. It represents the average data delay an application or a user experiences when transmitting data. The delay is usually measured in seconds.

Average end-to-end delay $=\underline{(\text { Total of Transmission Delays of All Received Packets })}$

Where, (Number of Packets Received)

Transmission Delay of a Packet $=($ Time Pkt. Rxvd. at Server - Time Pkt Txd. at Where 'time' is in seconds.

\section{$>$ Average Jitter:}

Jitter is a variation in packet transit delay caused by queuing, contention and serialization effects on the path through the network. In general, higher levels of jitter are more likely to occur on either slow or heavily congested links. The usual causes include connection timeouts, connection time lags, data traffic congestion, and interference. Simply put, this jitter is an undesirable output of system flaws and interruptions. Thus when jitters occur, computer monitors and computer processors may malfunction, files may get lost, downloaded audio files may acquire noise, Internet phone calls may get interrupted, suffer time lags or get disconnected.

Average Jitter $=\quad($ Total Packet Jitter for all Received Packets $)$

(Number of Packets Received - 1)

--- Eq 2.4

Where,

Packet Jitter $=($ Txn. Delay of Current Pkt - Txn. Delay of Previous Pkt $)$

Jitter can be calculated only if at least two packets have been received. 


\section{BER -- Bit Error Rate:}

Bit error occurs when one or more bits of data travelling across a network fail to reach their destination. Finite number of bits collectively forms a packet. Packet loss can be caused by a number of factors, including signal degradation over the network medium due to multi-path fading, packet drop because of channel congestion, corrupted packets rejected in-transit, faulty networking hardware, faulty network drivers or normal routing routines.

In addition to this, Bit Error probability is also affected by Signal-to-noise ratio and distance between the transmitter and receiver.

$\mathbf{B E R}=((\text { Packet transmitted }- \text { Packet received }) / \text { Session Time })^{*} 100 \quad$--- Eq 2.6

SNR-- Signal to Noise Ratio:

Signal-to-noise ratio is defined as the power ratio between a signal (meaningful information) and the background noise (unwanted signal):

$\mathbf{S N R}=\left[\mathrm{P}_{\text {signal }} / \mathrm{P}_{\text {Noise }}\right]$ Where $P$ is average power. Both signal and noise power must be measured at the same and equivalent points in a system, and within the same system bandwidth. If the signal and the noise are measured across the same impedance, then the SNR can be obtained by calculating the square of the amplitude ratio:

$\mathrm{SNR}=\left[\mathrm{P}_{\text {signal }} / \mathrm{P}_{\text {Noise }}\right]=\left(\mathrm{A}_{\text {signal }} / \mathrm{A}_{\text {Noise }}\right)^{2}$

where $A$ is root mean square (RMS) amplitude (for example, RMS voltage). Because many signals have a very wide dynamic range, SNRs are often expressed using the logarithmic decibel scale. In decibels, the SNR is defined as

$\mathrm{SNR}_{\mathrm{dB}}=10 \log 10\left(\mathrm{P}_{\text {signal }} / \mathrm{P}_{\text {Noise }}\right)=20 \log _{10}\left(\mathrm{~A}_{\text {signal }} / \mathrm{A}_{\text {Noise }}\right)$

\subsection{Contemporary VHO approaches}

Various vertical handover decision mechanisms have been proposed in literature based on various HO metrics and traffic classes (e.g., conversational, streaming, interactive, background) [19] - [22]. The handoff metrics and QoS parameters are categorized under different groups (e.g., bandwidth, latency, power, price, security, reliability, availability etc.). During the system discovery phase, mobile terminals equipped with multiple interfaces have to determine how many networks are available \& which types of services are available in each network. The networks may also advertise the supported data rates \& other QoS features for different applications. During the handoff decision phase, the mobile device determines which network it should connect toThe decision may depend on various parameters including the available bandwidth, delay, jitter, access cost, transmit power, current battery status of the mobile device, and the user's preferences. EVM proposed as Generic-QoS trigger for VHO which is in good agreement to characterise a radio link and possibly optimise VHO execution, the final phase.[23] [24]

\section{Hybrid ANP (Analytic Network Process)}

It is used only for weight elicitation.

\section{ANP algorithm:}

1. Formulate the Problem

2. Prepare Criteria/ Attributes 
3. Alternatives

4. Determine Relative Weight of Criteria's using Fuzzy rule

5. Determine Weight of Each Criteria Using Eigenvector Method

6. Obtain Weight Matrix, W [25].

\section{Modified RTOPSIS}

RTOPSIS is an acronym for Rank reversal Technique for Order Preference by Similarity to Ideal Solution. This method is used to calculate the cost function and accordingly rank the network.

\section{RTOPSIS algorithm:}

1. Construct the Decision Matrix, D With the Values Taken from Qualnet

2. Obtain Cost Function, minimum the better

3. Construct Normalized Decision Matrix, $\mathrm{R}$

$$
r_{s i}=s_{i} / \sqrt{\sum_{i=1}^{m} s_{i}^{2}}
$$

Where $\mathrm{x}_{\mathrm{ij}}=$ elements of decision matrix ,D

4. Calculate Weight Normalized Decision Matrix, $\mathrm{V}=[\mathrm{W}] *[\mathrm{R}]$

5. Determine $+v e$ and - ve Ideal Solution $A^{+}=\operatorname{MAX}(V), A^{-}=\operatorname{MIN}(\mathrm{V})$

6. Calculate Separation Measure

$$
S_{i}^{+}=\sqrt{\sum_{i=1}^{n} \frac{\left(A_{i}^{+}-v_{i j}\right)^{2}}{w_{i}}}, S_{i}^{-}=\sqrt{\sum_{j=1}^{n} \frac{\left(A_{j}-v_{i j}\right)^{2}}{w_{i}}}
$$

7. Calculate Cost : $\mathrm{C}=\mathrm{S}^{-} /\left(\mathrm{S}^{-}+\mathrm{S}^{+}\right)$

8. Rank the network on the basis of cost function [26][ 27]

\section{Modified Oliver blume method}

This method proposes network selection mechanism based on quality of service parameters. At first Interworking environment is designed on Qualnet. Applying different traffic types, QoS parameters are observed, called 'QoS determine'. QoS level desired by the receiver/user for the existing application is represented by 'QoS application' weighted ratio of all QoS triggers are used to calculate Cost function of each network. Blume approach can be summarised as follows:

\section{Blume algorithm:}

1. Construct Weight Matrix for Attributes Based on Literature Survey, W

2. Take observation from Qualnet simulation for different traffic class

3. Construct the Decision Matrix, D

4. Construct the Ideal Matrix 'I' consisting of $\mathrm{min} / \mathrm{max}$ value of attribute for each network

5. Calculate Cost Function, $\mathrm{C}=\prod\left(\mathrm{QoS}_{\text {determine }} / \mathrm{QoS} \text { application }\right)^{\text {weights }}$

6. Calculate $\mathbf{C}_{\mathbf{i}}=\prod_{\mathbf{i}}\left(\mathbf{D}_{\mathbf{i j}} / \mathbf{I}_{\mathbf{i j}}\right)^{\mathbf{W}_{\mathbf{i j}}}, \mathbf{w}_{\mathbf{i j}}$ is weightage of QoS trigger w.r.t application

7. Apply Clipping Function : $\operatorname{CLIP}_{\mathbf{i}}\left(\mathbf{D}_{\mathbf{i j}} / \mathbf{I}_{\mathbf{i j}}\right)=1$ for $\left(\mathbf{D}_{\mathbf{i j}} / \mathbf{I}_{\mathbf{i j}}\right)=0$

8. Rank the Network with Minimum Cost [28] 
VHO decision is based on the value of cost function obtained by the above mentioned methods. In both methods, Assignment of weightage to all criteria is a very critical task i.e. which criteria are bearing more importance w.r.t. which traffic type. Next step is to obtain a relative weight matrix in which weights are assigned with respect to each other which are at the same level i.e. which attribute is more important? And how strongly related/dependent? A fuzzy based fundamental 1-9 Scale is used typically. [Refer table-2] Say BER is compared against avg jitter i.e. BER is $1 / 3$ times important as compared to Jitter, which means BER is 3 times less important than Jitter, where as when compared with throughput it is $5 / 3$ times, or alternatively it is 5 times more important when compared with Jitter $\left(5^{*} 1 / 3\right)$. A result matrix is set up after a series of comparisons. See section 3.2 for details.

Cost function is a function that defines the cost of a network w.r.t. QoS parameters i.e. Cost $=\mathrm{f}$ ( QoS determine, QoS application, weights) Where 'QoS determine' is the observed QoS parameters and 'QoS application' is the corresponding QoS parameter as requested by an application running on the terminal device. Weight is the weightage of each QoS parameter w.r.t. to application, Network with minimum cost function is the best for a given application. Decision metrics' are same for all the three methods, modified ANP, RTOPSIS and Blume method which are obtain using the Qualnet 5.0 Test Beds. [29] [30]

\section{Modelling OF NeTwORK COST FOR VHO DeCISION 3.1 Proposed analytical model (Problem Formulation)}

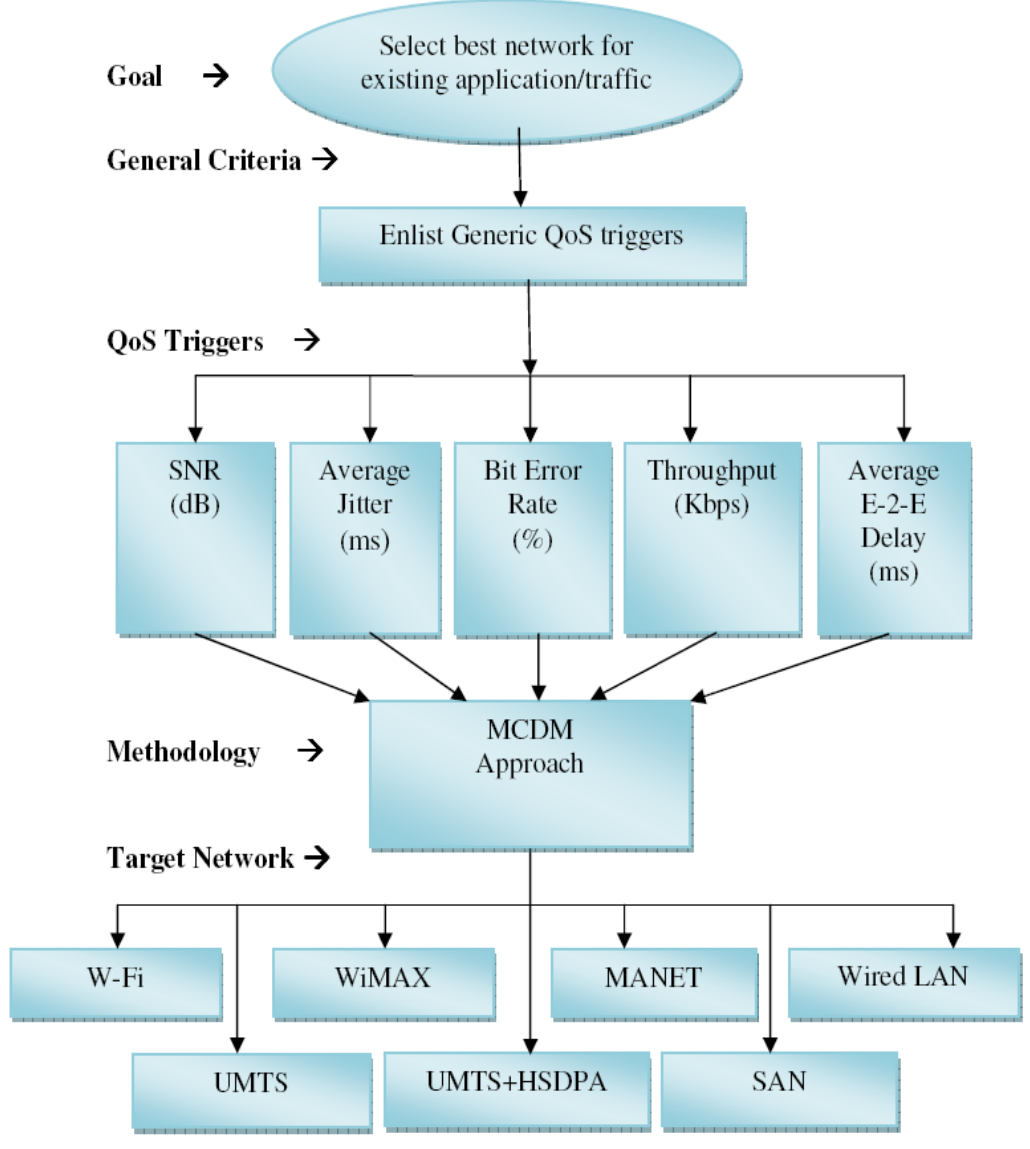




\subsection{MCDM weight assignment technique (neuro-fuzzy based)}

Decision making with more than one criterion is called Multi Criteria Decision Making (MCDM) [28]. Network selection is a key issue in converged heterogeneous networking environment. A traditional way to select a target network is only based on the received signal strength (RSS or also called SNR); however, it is not comprehensive enough to meet the various demands of different multimedia applications and different users. The proposed schemes have considered multiple criteria (e.g. Delay, Jitter, BER, Throughput etc.) for access network selection [31].

Table- 2

\begin{tabular}{|c|c|c|c|c|c|c|}
\hline QoS triggers & $\begin{array}{c}\text { Avg. E2E } \\
\text { delay (ms) }\end{array}$ & $\begin{array}{c}\text { Avg. Jitter } \\
(\mathrm{ms})\end{array}$ & BER & Throughput & SNR & Weight \\
\hline Avg E-E Delay & 1 & 1 & 3 & 5 & 4 & 0.3309 \\
\hline Avg Jitter & 1 & 1 & 3 & 5 & 4 & 0.3309 \\
\hline BER & $1 / 3$ & $1 / 3$ & 1 & $5 / 3$ & $4 / 3$ & 0.1103 \\
\hline Throughput & $1 / 5$ & $1 / 5$ & $3 / 5$ & 1 & $4 / 5$ & 0.0662 \\
\hline SNR & $1 / 4$ & $1 / 4$ & $4 / 3$ & $4 / 5$ & 1 & 0.0772 \\
\hline
\end{tabular}

All above matrices are inconsistent because all rows are not multiples of each other. When we encounter this kind of matrix, the most commonly used method is eigenvalue /eigenvector method.

The number of nonzero eigenvalues for a matrix is equal to its rank.

A consistent matrix has rank 1 , so as the number of its eigenvalues, and an inconsistent matrix typically has more than 1 eigenvalue.

We use the largest, $\lambda_{\max }$, for consistency measurement computation.

Knowing, $\mathrm{A}=[1,3,5 ; 1 / 3,1,13 ; 1 / 5,1 / 3,1]$

we can calculate eigenvalue $\lambda$ by solving, $\operatorname{det}(\lambda \mathrm{I}-\mathrm{A})=0$

The maximum is adopted for calculations, the normalized vector $\mathbf{w}$ of weights can be obtained from the formula $\mathbf{A} \mathbf{w}=\lambda_{\max } \mathbf{w}$

Here,

$$
\begin{aligned}
& \mathbf{w}_{\mathbf{c}} \quad=\quad \text { Initial Weight of Attributes for Conversational Traffic (Table 8.3) } \\
& =\quad\left\{\mathrm{w}_{\mathrm{c}(\text { Delay })}, \mathrm{W}_{\mathrm{c}(\mathrm{Jitter}),} \mathrm{W}_{\mathrm{c}(\mathrm{PLR}),} \mathrm{W}_{\mathrm{c}(\text { Throughput }),} \mathrm{W}_{\mathrm{c}(\mathrm{EVM})}, \mathrm{w}_{\mathrm{c}(\mathrm{SNR})}\right\}
\end{aligned}
$$

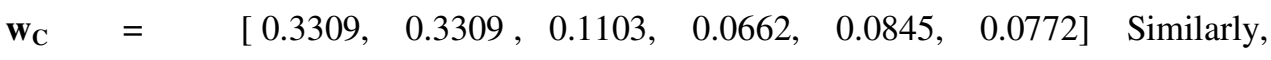

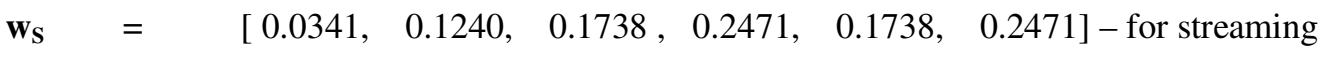

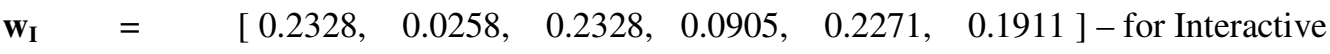

$$
\begin{aligned}
& \mathbf{w}_{\mathbf{B}}=\left[\begin{array}{llll}
0.0317, & 0.0317, & 0.2122, & 0.2996,0.1973,0.2274
\end{array}\right]-\text { for Background }
\end{aligned}
$$




\subsection{Traffic-wise performance sheet obtained from Qualnet}

Table- 3

\begin{tabular}{|c|c|c|c|c|c|c|c|}
\hline $\begin{array}{l}\text { Wireless } \\
\text { Network }\end{array}$ & Traffic Class & Application & $\begin{array}{l}\text { Session time } \\
\text { (SEC) }\end{array}$ & $\begin{array}{l}\text { Avg E2E } \\
\text { delay (ms) }\end{array}$ & $\begin{array}{l}\text { Jitter } \\
\text { (ms) }\end{array}$ & $\begin{array}{l}\text { Bit } \\
\text { Error } \\
\text { Rate - \% }\end{array}$ & $\begin{array}{l}\text { Throughput } \\
\text { (kbps) }\end{array}$ \\
\hline \multirow[t]{4}{*}{802.11} & Conversational & VOIP & 92.0637 & 44.519 & 47.388 & 0.0701 & 31.468 \\
\hline & Streaming & CBR & 23.0003 & 9.6282 & 0.1041 & 0 & 4.274 \\
\hline & Interactive & MCBR & 22.9976 & 9.6282 & 0.313 & 0 & 4.272 \\
\hline & Background & FTP & 57.2106 & 5.0001 & 2.6302 & 0 & 814.753 \\
\hline \multirow[t]{4}{*}{802.16} & Conversational & VOIP & 238.9102 & 46.63425 & 47.9016 & 2.6139 & 7.173 \\
\hline & Streaming & CBR & 22.9965 & 9.4255 & 0.1103 & 0 & 4.274 \\
\hline & Interactive & MCBR & 23.0 & 9.4254 & 10.0174 & 0 & 4.272 \\
\hline & Background & FTP & 35.2832 & 5.3041 & 6.2009 & 0 & 807.755 \\
\hline \multirow[t]{4}{*}{ MANET } & Conversational & VOIP & 237.5264 & 107.469 & 0 & 0.3816 & 22.6712 \\
\hline & Streaming & CBR & 22.7662 & 101.711 & 7.8634 & 0 & 4.317 \\
\hline & Interactive & MCBR & 22.7662 & 101.711 & 217.982 & 0 & 4.312 \\
\hline & Background & FTP & 135.9578 & 19.3933 & 81.0155 & 0.0036 & 96.166 \\
\hline \multirow[t]{4}{*}{ SAN } & Conversational & VOIP & 233.7556 & 325.7 & 0.7016 & 0 & 12.085 \\
\hline & Streaming & CBR & 23.00 & 280.926 & 0.1125 & 0 & 4.274 \\
\hline & Interactive & MCBR & 23.2807 & 280.926 & 292.904 & 0 & 4.272 \\
\hline & Background & FTP & 202.8639 & 2.2283 & 1.0000 & 0.0612 & 230.705 \\
\hline \multirow[t]{4}{*}{ UMTS } & Conversational & VOIP & 172.2791 & 1787.37 & 31445.2 & 0 & 22.7649 \\
\hline & Streaming & CBR & 18.405 & 412.273 & 22.0182 & 16.667 & 4.442 \\
\hline & Interactive & MCBR & 18.440 & 142.705 & 2518.68 & 16.667 & 4.440 \\
\hline & Background & FTP & 290.4743 & 8.0321 & 493.914 & 0.2401 & 58.300 \\
\hline \multirow{4}{*}{$\begin{array}{l}\text { UMTS+ } \\
\text { HSDPA }\end{array}$} & Conversational & VOIP & 173.5511 & 69.1163 & 25562.8 & 2.0288 & 18.5195 \\
\hline & Streaming & CBR & 18.4405 & 412.273 & 22.0182 & 16.667 & 4.442 \\
\hline & Interactive & MCBR & 18.4404 & 142.705 & 251.873 & 16.667 & 4.440 \\
\hline & Background & FTP & 290.474 & 8.7586 & 493.965 & 0.7401 & 58.300 \\
\hline \multirow{4}{*}{$\begin{array}{l}802.3 \\
\text { WIRED } \\
\text { LAN }\end{array}$} & Conversational & VOIP & 174.0927 & 71.150 & 6991.01 & 6.2167 & 32.2699 \\
\hline & Streaming & CBR & 28.098 & 90.345 & 22.134 & 4.286 & 5.628 \\
\hline & Interactive & MCBR & 27.456 & 89.234 & 240.92 & 2.456 & 4.976 \\
\hline & Background & FTP & 138.982 & 14.342 & 50.456 & 0.9872 & 900.234 \\
\hline
\end{tabular}

For details refer [28]-[31]. 


\section{NGN TESTBEDS / INTERWORKING SCENARIOS}

\subsection{Qualnet Simulation}

All the technologies must be compatible to operate together for successful seamless vertical handover .To test the compatibility and to find the attribute values for underlying network, we have designed seven test beds with the help of Qualnet 5.0 on the basis of model in fig. 3 to 6 .

$>$ Subscriber starts using an application under Wi-Fi access.

$>$ After a while, user leaves home for work and moves towards another Wi-Fi covered area.

$>$ In between the application is supported by a $3^{\text {rd }}$ network like Wi-Fi, WiMAX, MANET, UMTS, UMTS+HSDPA, Wired LAN.

Qualnet enables users to design new protocol models, Optimize new and existing models, Design complex wireless networks using user-designed models, analyze the performance of networks and helps in advanced networking planning. Qualnet 5.0 GUI consists of Architect, Analyzer, Packet Tracer, and File Editor [29]. These modes are accessible from the Components Toolbar see fig- 2.

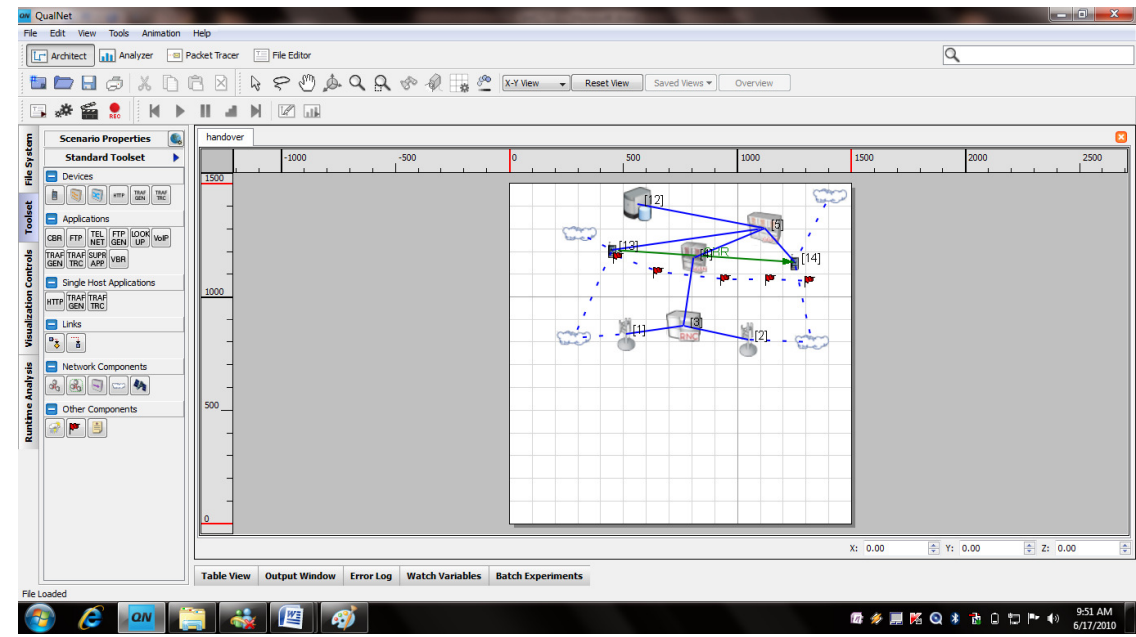

Figure- 2: Qualnet Design architect

\subsection{NGN Testbeds / Interworking Scenarios}

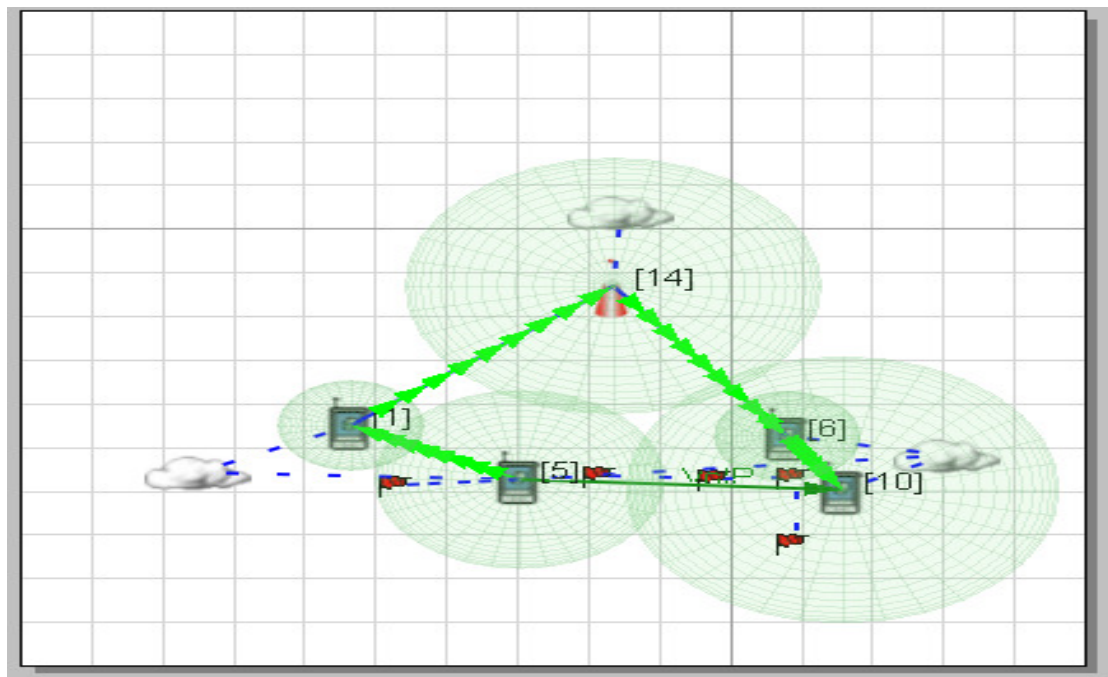

Figure- 3 Wi-Fi $\rightarrow$ WiMax $\rightarrow$ Wi-Fi 


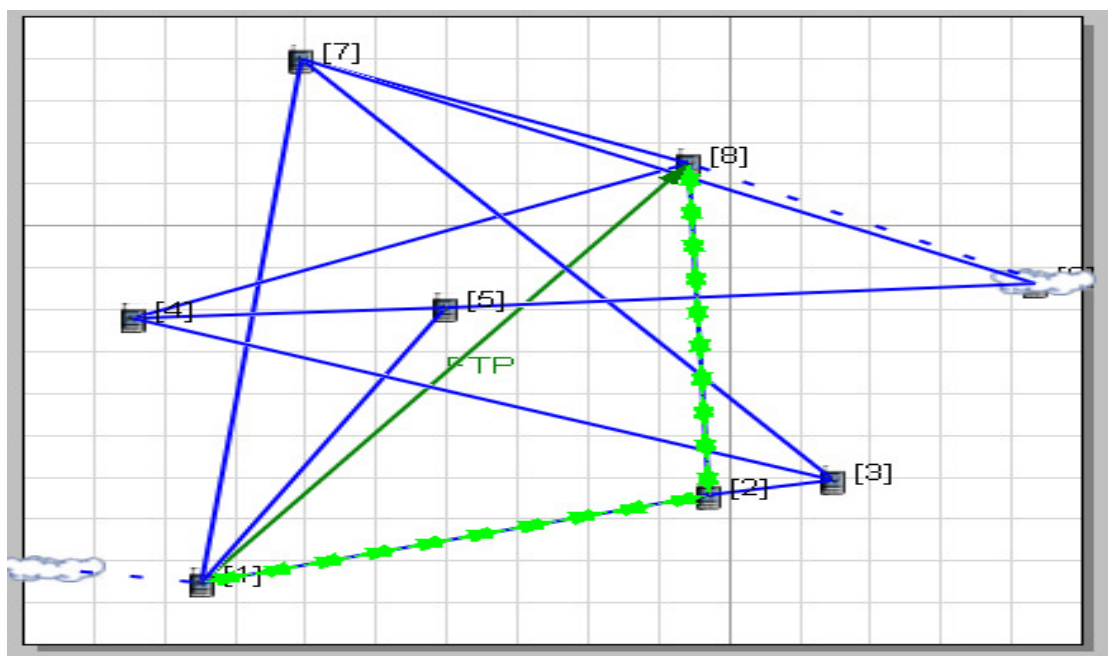

Figure- 4 Wi-Fi $\rightarrow$ MANET $\rightarrow$ Wi-Fi

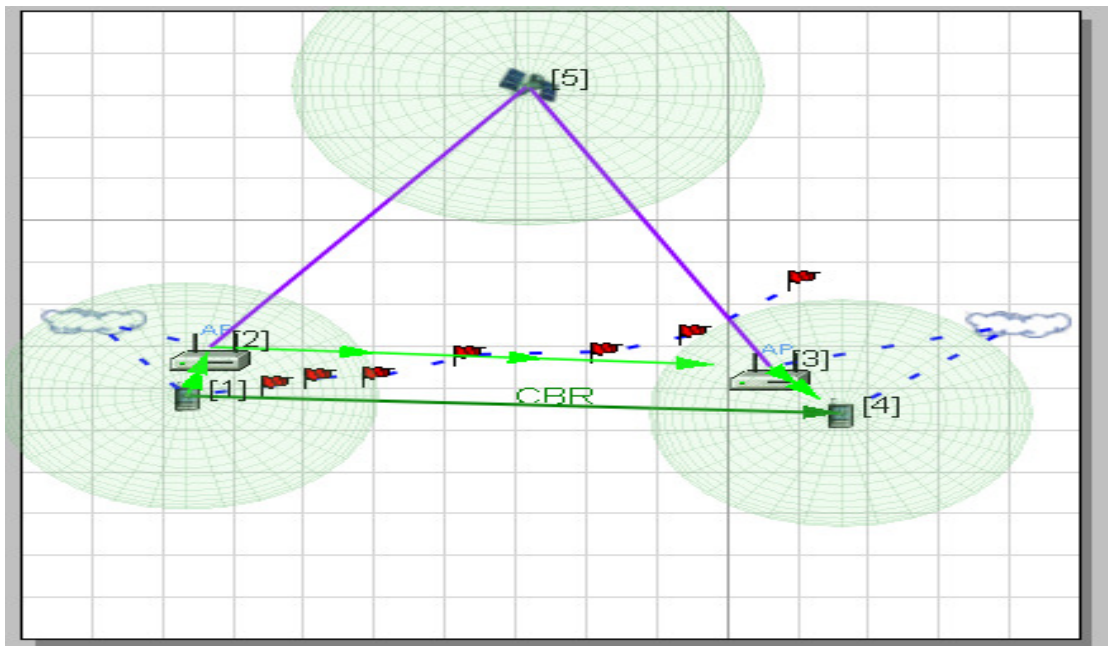

Figure- 5 Wi-Fi $\rightarrow$ Satellite $\rightarrow$ Wi-Fi

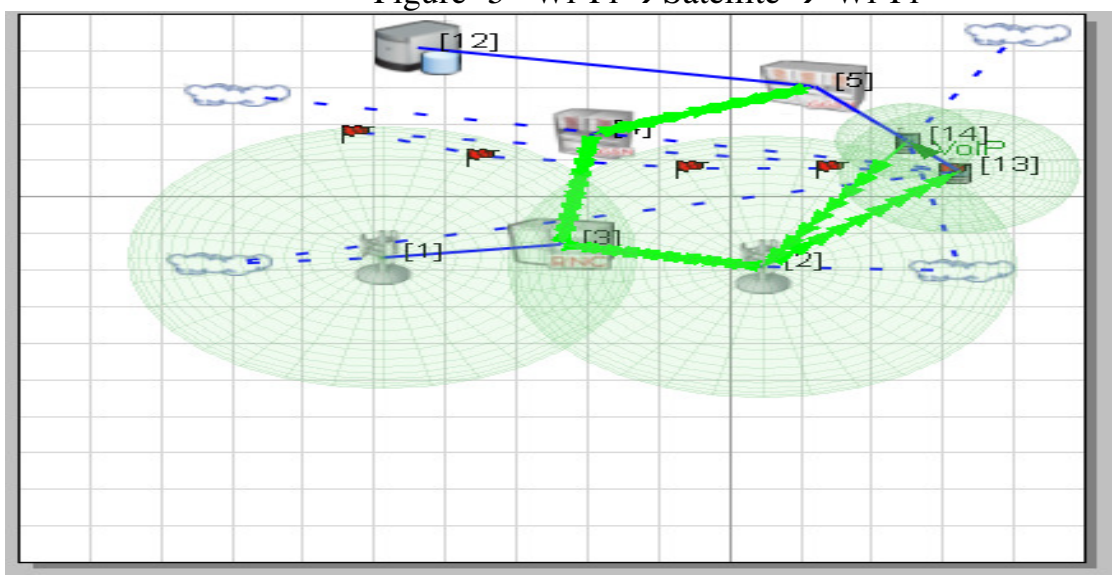

Figure- 6 Wi-Fi $\rightarrow$ UMTS $\rightarrow$ Wi-Fi 


\section{Performance Analysis}

\subsection{Simulated Results and Plots}

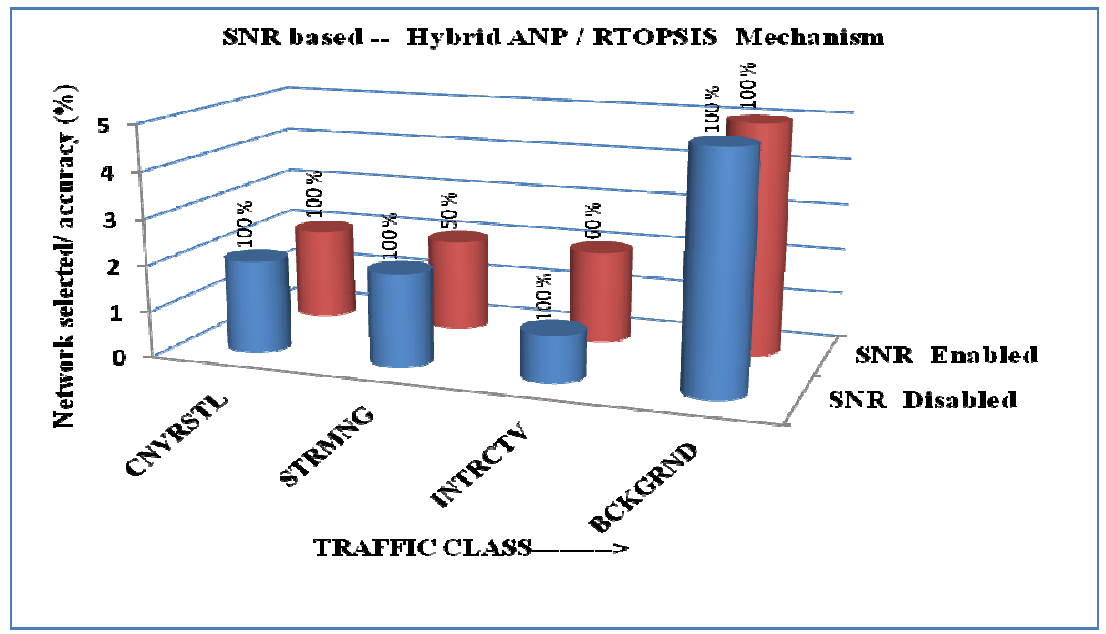

Figure- 7

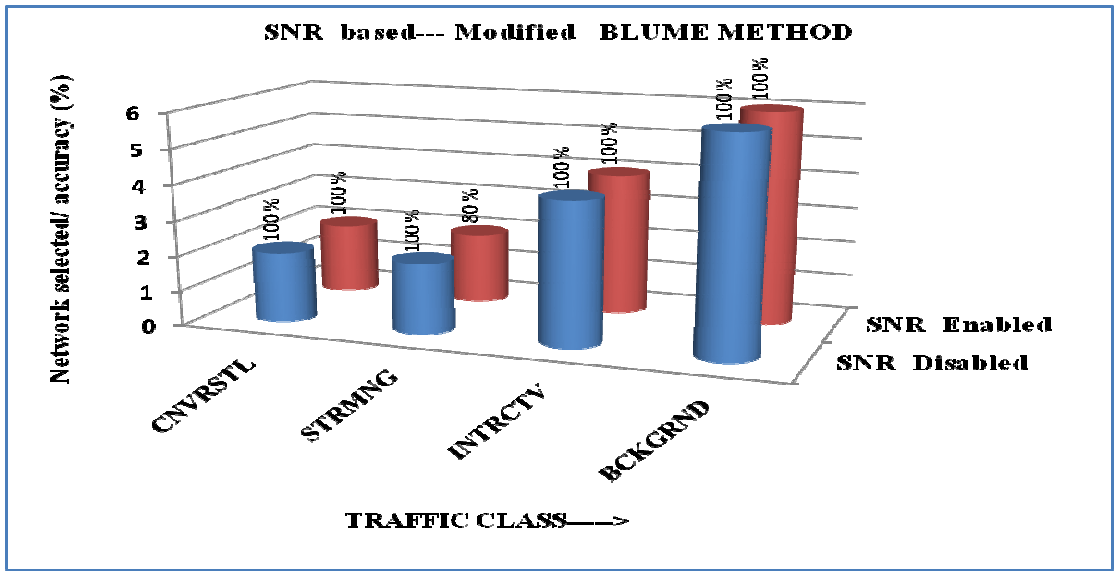

Figure- 8

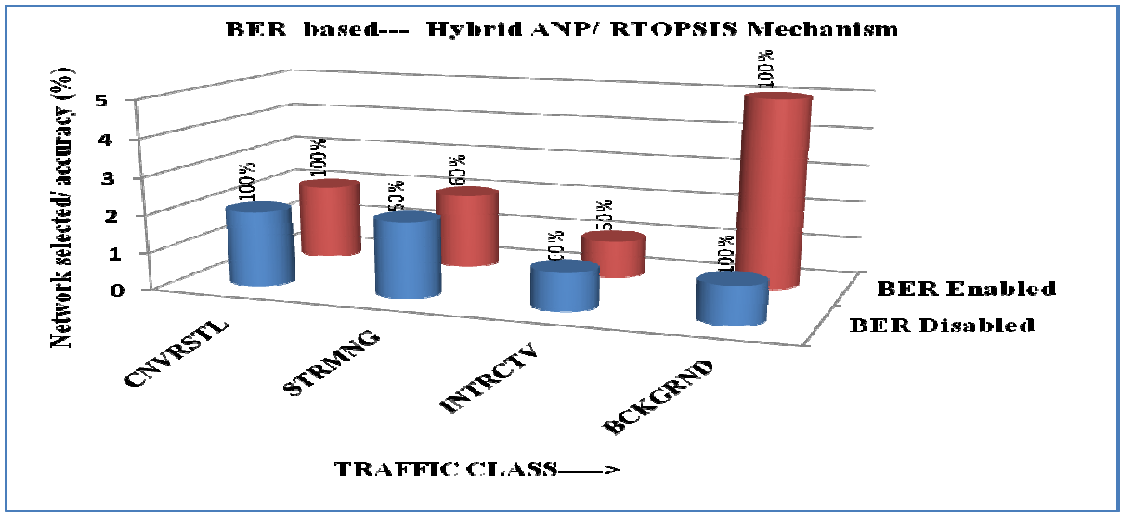

Figure- 9 


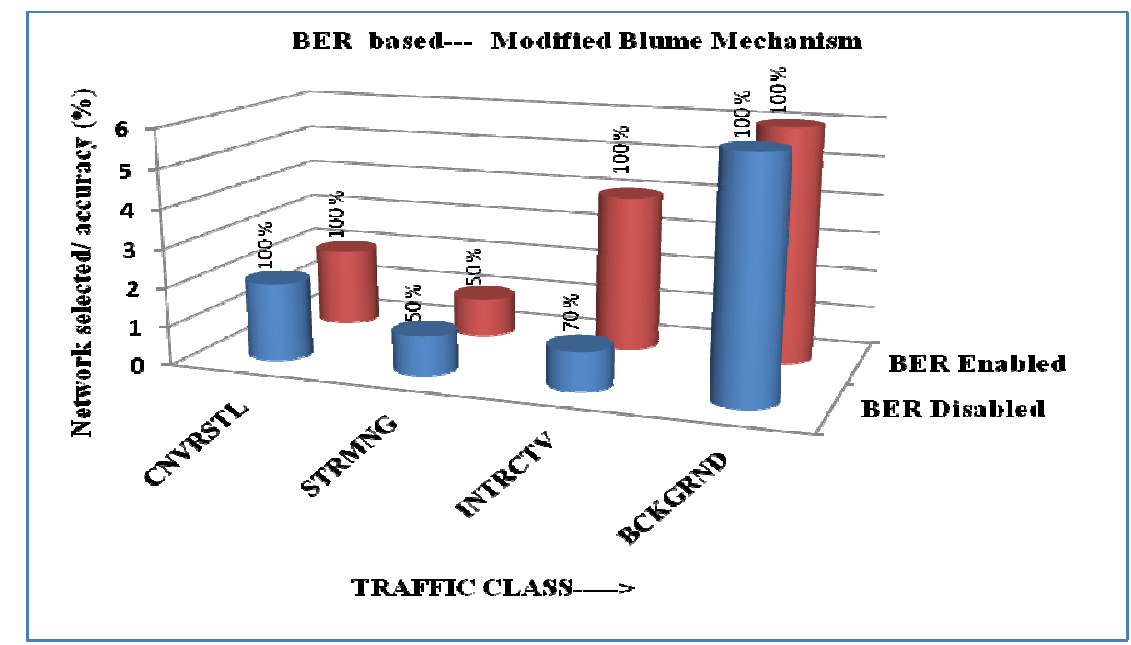

Figure- 10

\begin{tabular}{|c|c|c|c|c|c|c|}
\hline $\mathbf{1}$ & $\mathbf{2}$ & $\mathbf{3}$ & $\mathbf{4}$ & $\mathbf{5}$ & $\mathbf{6}$ & $\mathbf{7}$ \\
\hline Wi-Fi & WiMAX & MANET & LAN & SAN & UMTS & UMTS+HSDPA \\
\hline
\end{tabular}

\subsection{Comparison of VHO mechanisms}

Table- 5.1

\begin{tabular}{|c|c|c|c|c|c|c|}
\hline \multicolumn{2}{|c|}{ Traffic/ Mechanism } & \multicolumn{2}{|c|}{ CONV } & STRM & INTR & BACK \\
\hline \multicolumn{2}{|c|}{ ANP_SNR } & \multicolumn{2}{|c|}{2} & 2 & 1 & 5 \\
\hline \multicolumn{2}{|c|}{ BLUME_SNR } & \multicolumn{2}{|c|}{2} & 2 & 4 & 6 \\
\hline \multicolumn{2}{|c|}{ ANP_BER } & \multicolumn{2}{|c|}{2} & 2 & $1 / 2$ & $1 / 5$ \\
\hline \multicolumn{2}{|c|}{ BLUME_BER } & \multicolumn{2}{|c|}{2} & 1 & 4 & 6 \\
\hline 1 & 2 & $\mathbf{3}$ & 4 & 5 & 6 & 7 \\
\hline Wi-Fi & WiMAX & MANET & LAN & SAN & UMTS & UMTS+HSDPA \\
\hline
\end{tabular}

Table- 5.2

\begin{tabular}{|c|c|c|c|}
\hline & Mechanism & CPU Time & Good processor \\
\hline SNR based & Hybrid ANP/RTOPSIS & $128.7161 \mathrm{sec}$ & $1.28 \mathrm{sec}$ \\
\hline SNR based & Modified BLUME & $6.9467 \mathrm{sec}$ & $69.4 \mathrm{msec}$ \\
\hline BER based & $\begin{array}{c}\text { Modified Hybrid } \\
\text { ANP,RTOPSIS }\end{array}$ & $145.4453 \mathrm{sec}$ & $1.44 \mathrm{sec}$ \\
\hline BER based & $\begin{array}{c}\text { Modified } \\
\text { BLUME }\end{array}$ & $7.9928 \mathrm{sec}$ & $79.9 \mathrm{msec}$ \\
\hline
\end{tabular}




\subsection{Elucidation of Results:}

Based on the algorithm available in the literature, both the VHO mechanisms are implemented. Here at first Different Interworking Testbeds were designed. Then observed various important QoS triggers which characterises a given network like SNR, BER, Throughput, Jitter, End to end delay. Practical attributes are needed for the parameters which are used simulate the analytical model. These attributes are obtained by running the scenarios on Qualnet. Then traffic-class-wise a performance sheet is prepared [table-1]. These values are used as input parameters to model ANP and Blume approaches. Model was iterated 10 times and the average value for weights, cost function was iterated 25 times to get a consistent value.

$>$ While simulating the VHO models, all five weighted triggers are used i.e. weighted combination of SNR, BER, Throughput, Jitter, End to end delay triggers [section $2.3 \& 4$ ] to calculate a final network cost. Based on this cost function, network is selected. Paper investigates effect of SNR and BER on network selection i.e. which network is selected during $\mathrm{VHO}$ for desired traffic class (conversational, streaming, interactive or background).

$>$ In figure 7, Effect of SNR is analysed, when enabled and disabled in the hybrid ANP mechanism. In collocated environment of seven types of networks, for interactive application, user prefers to switch to network 1 when SNR is disabled \& to network 2 when enabled. This implies if we consider SNR for VHO decision along with other QoS trigger then N1 [Wi-Fi] is the best network with $100 \%$ accuracy. When SNR is disabled then N2 [WiMAX] is selected with $60 \%$ accuracy. Similarly for other traffic classes, selected best network is different.

$>$ Accuracy is used in paper to describe the performance of VHO decision. Accuracy is defined as the ratio (\%) of number of times a given network is selected to total number of VHO trials. i.e. $60 \%$ accuracy of network N2 [WiMAX] means when VHO process was executed / attempted 25 times, 15 times N2 is selected. Since 15 times N2 is getting selected thus it gives an accuracy of making a VHO decision (consistently) for target network selection.

$>$ Similarly in figure-8, effect of SNR is observed for modified Blume mechanism. For the same interactive traffic type, N3 [MANET] is selected with 100\% accuracy.

$>$ In figure 9, we observe the inclusion/exclusion of BER in computing the cost function of the collocated networks. For the same interactive traffic class, N1 [Wi-Fi] is rated the best with $50 \%$ accuracy for hybrid ANP, where as it is N4 [wired LAN] for modified Blume mechanism [figure 10].

$>$ Table- 5.1 summarises the overall result i.e. which network is selected the best with maximum accuracy.

$>$ Table 5.2 provides an insight to computational time for executing the said MCDM approach. With a good processor this CPU time can be greatly reduced which can significantly reduced the VHO latency.

$>$ In figure $7 \& 8$, there is negligible change in Network selection when SNR is incorporated therefore in case of co-existing wireless environment in Blume's method, SNR can be excluded. 
The analysis revealed that the modified Blume approach is giving more stable result in comparison of modified ANP and RTOPSIS approach because it is selecting the target network with higher accuracy/ consistency.

\section{CONCLUSION}

Paper explores the QoS based multi criteria-approach to initiate a vertical handover. Paper investigates both the contemporary VHO mechanism hybrid ANP and modified Blume approach. The performance analysis of the mechanisms suggests that modified blume seems to be a more promising VHO mechanism because it is less complex i.e. it is simple to implement, more efficient (40\% more accurate), quickly computed (20 times less) and more dynamic (application oriented). With Blume approach we may incorporate other QoS metrics and can further optimise the Network selection process. The work is being carried to accommodate Long Term EvolutionLTE (emerging 3GPP-2 standard) as another candidate network for VHO execution. In future scope of the paper, VHO utility function may be developed to comment on profitability of VHO Decision. With the proposed testbeds, Mobility management may be further expedited for Next Generation Network.

\section{REFERENCES}

[1] Lu, Guang, 'Enable multimedia mobility with IEEE 802.21', World of Wireless Mobile and Multimedia Networks (WoWMoM), 2010 IEEE International Symposium , DOI: 10.1109/WOWMOM.2010.5534987, Page(s): 1 - 6, Publication Year: 2010

[2] Lei Shi,Changbin Liu, Bin Liu, "Network utility maximization for triple-play services", Science Direct, Elsevier, Computer Communications 31 (2008) 2257-2269, 2008.

[3] J. McNair and F. Zhu, "Vertical Handoffs in Fourth-generation Multinetwork Environments," IEEE Wireless Comm., vol. 11, no. 3, June 2004.

[4] 3GPP, "Requirements on 3GPP System to Wireless Local Area Network (WLAN) interworking," TS 22.234 (v7.0.0), January 2005.

[5] Xiaoyu Liu, Youn-Hee Han, "Handover Scenarios and Use Cases between 802.16 and 802.11" ,IEEE , 2004.

[6] Rajender Kumar and Brahmjit Singh, 'EVM as generic QoS trigger for Heterogeneous wireless overlay networks', International Journal of Wireless \& Mobile Networks ( IJWMN ), AIRCCS, DOI : 10.5121/ijwmn.2010.2315 Volume-2, no.3, Page(s): 214 - 225, Publication Year : August 2010

[7] AUKBC Research center presentation , "Mobile WiMAX", 2008.

[8] www.umtsworld.com/technology/overview.htm

[9] Reena Sharma, Rajender Kumar, Brahmjit Singh, "Modulation Error as QoS Trigger in Emerging MANET Networks for Optimum Vertical Handover: Measurement Report”, CIET Punjab, International conference on wireless network proceeding, 2008.

[10] Andrea Calvagna, Aurelio La Corte ,Sabrina Sicari, "Mobility and quality of service across heterogeneous wireless networks", Science Direct, Elsevier, Computer Networks 47 (2005) 203-217, 2005 .

[11] Niswar, M.; Kashihara, S.; Taenaka, Y.; Tsukamoto, K.; Kadobayashi, Y.; Yamaguchi, S.; Seamless vertical handover management for VoIP over intermingled IEEE 802.11g and IEEE 802.16e , Information and Telecommunication Technologies (APSITT), 2010 8th Asia-Pacific Symposium , Page(s): 1 - 6, Publication Year: 2010 
[12] Wei-wei Jiang, Hong-yan, Cui Qiang-jun, Van Xiao-juan, Wang Jian-Ya Chen, “A Novel ApplicationOriented Dynamic Network Selection in an Integrated UMTS and WiMAX Environment”, ,IEEE.2008.

[13] Olga Ormond, "Economic Model for Cost Effective Network Selection Strategy in Service Oriented Heterogeneous Wireless Network Environment", IEEE.2006.

[14] Feng He, Furong Wang, "A cost sensitive best network selection scheme in heterogeneous wireless networks", 1753-1756,IEEE. 2007.

[15] Wei Shen, Qing-An Zeng, "Cost-Function-Based Network Selection Strategy in Integrated Wireless and Mobile Networks" , IEEE Transactions on Vehicular Technology, VOL. 57, NO. 6, 3778-3788, November 2008 .

[16] Oliver Blume, Method of Identifying a radio link, Inventor: Oliver, Stuttgart (DE), patent US 7,616, 928 B2, date of patent Nov. 10, 2009.

[17] Choi, H.-H.; Song, O.; Park, Y.-K.; Lee, J.-R.,'Performance Evaluation of Opportunistic Vertical Handover Considering On-Off Characteristics of VoIP Traffic', Vehicular Technology, IEEE Transactions , Volume: 59, Issue: 6, DOI: 10.1109/TVT.2010.2049510 , Page(s): 3115 - 3121, Publication Year: 2010.

[18] Leijia Wu; Sabbagh, A.; Sandrasegaran, K.; Elkashlan, M.; Cheng-Chung Lin; Performance Evaluation on Common Radio Resource Management Algorithms, Advanced Information Networking and Applications Workshops (WAINA), 2010 IEEE 24th International Conference , DOI: 10.1109/WAINA.2010.13 , Page(s): 491 - 495, Publication Year: 2010.

[19] Trent E. LANGE, "Simulation of heterogeneous neural networks on serial and parallel machines", Science Direct, Parallel Computing 14 (1990) 287-303 287 North-Holland, 1990.

[20] Thumthawatworn, T.; Pervez, A.,' Multi-level rule-based handover framework for heterogeneous wireless networks 'Wireless Advanced (WiAD), 2010 6th Conference, DOI: 10.1109/WIAD.2010.5544876 , Page(s): 1 - 6, Publication Year: 2010.

[21] Chantaksinopas, I.; Oothongsap, P.; Prayote, A.,' Framework for network selection transparency on vehicular networks ' Electrical Engineering/Electronics Computer Telecommunications and Information Technology (ECTI-CON), 2010 International Conference, Page(s): 593 - 597, Publication Year : 2010.

[22] Inwhee Joe; MinChul Shin, 'A Mobility-Based Prediction Algorithm with Dynamic LGD Triggering for Vertical Handover ,Consumer Communications and Networking Conference (CCNC), 2010 7th IEEE , DOI: 10.1109/CCNC.2010.5421627, Page(s): 1 - 2, Publication Year: 2010.

[23] Patent no. US 7,613,457 B2; Inventors: Xia Gao, Campbell, CA (US); Gang Wu, Cupertino, CA(US)systems and method for supporting Quality of Service in vertical handovers between heterogeneous networks, , date of patent-Nov. 3, 2009.

[24] Eng Hwee Ong; Khan, J.Y, On optimal network selection in a dynamic multi-RAT environment , Communications Letters, IEEE Volume: 14 , Issue: 3 , DOI: 10.1109/LCOMM.2010.03.092378, Page(s): 217 - 219, Publication Year: 2010.

[25] J. S. Dyer, -Remarks on the Analytic Hierarchy Process, Management Science, Volume 36 Number 3, Page 249-258, 1990.

[26] C. Carlsson, R. Fuller, -Fuzzy Multiple Criteria Decision Making: Recent Developments, Fuzzy Sets and Systems, Volume 78, Number 2, Page 139-153, March 1996.

[27] F. Bari, and V. C. M. Leung, -Multi-Attribute Network Selection by Iterative TOPSIS for Heterogeneous Wireless Access, in Proc. IEEE CCNC'07 Las Vegas, NV, USA, January 2007.

[28] Z. Ozturk, -A Review of Multi Criteria Decision Making with Dependency between Criteria, in Proc. of MCDM 2006, Chania, Greece, June 2006. 
[29] Qualnet 5.0 Simulator User Guide, www.scalable-network.com.

[30] Vulpe, A.; Obreja, S.G.; Barbu, O.-E.; Penciu, C.-D.; Bugă, M.; Ciobotaru, B., 'QualNet implementation for mobility management in a MIH enabled system , Communications (COMM), 2010 8th International Conference, DOI: 10.1109/ ICCOMM.2010.5509048, Page(s): 523 - 526, Publication Year: 2010 .

[31] W. Zhang, - Handover Decision Using Fuzzy MADM in Heterogeneous Networks, in Proc. of IEEE WCNC'04, Atlanta, GA, USA, March 2004.

[32] Alessio Botta, Antonio Pescape',Giorgio Ventre, "Quality of service statistics over heterogeneous networks: Analysis and applications" ,Science Direct, Elsevier, European Journal of Operational Research 191 (2008) 1075-1088, 2008.

[33] RFC 791, RFC 2474.

\section{Author's Profile}

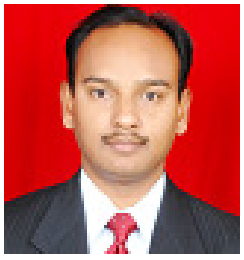

Rajender Kumar, Asstt. Professor

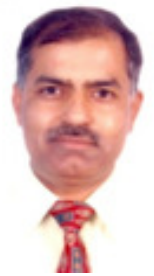

Dr. Brahmjit Singh, Professor

\section{Corresponding Author Biography: Rajender Kumar}

Rajender Kumar is working as Asstt. Professor in Electronics and Communication Engineering Department at NIT Kurukshetra. He is pursuing PhD in Vertical Handovers (Heterogeneous Wireless Networks). He did B.Tech in Electronics and Communication Engineering (ECE) from Institute of Engineering Technology, Kanpur in 2001. He received M.Tech (Hons.) in ECE from National Institute of Technology, Kurukshetra, (INDIA) in 2004. He worked in Indian Telephone Industries as NSS (switch) Engineer at Pune, in GSM (Mobile) Division. He has 6+ years of teaching/ industrial and research experience. He teaches Telecommunication networks, Statistical modeling, Mathematical models for internet and web in M.Tech program. He takes Computer communication networks and Signals \& system in B.Tech program. He has 18 research paper publications in National and International Conferences and Journal. His field of Interest includes Vertical Handovers, Advanced Wireless Systems, Cognitive radio, NGWN challenges \& Cross Layer Design Issues. He is reviewer and consultant of leading publisher and government organization like Tata Mc-Graw Hill publishers \& KDB respectively. Rajender Kumar received 'All India Young Scientist Award' in Silver Jubilee function held at Bhopal in November, 2007.

\section{Co-Author Biography: Brahmjit Singh}

Brahmjit Singh received B.E. degree in Electronics Engineering from Malaviya National Institute of Technology, Jaipur in 1988, M.E. degree in Electronics and Communication Engineering from Indian Institute of Technology, Roorkee in 1995 and $\mathrm{PhD}$ degree from Guru Gobind Singh Indraprastha University, Delhi in 2005 (INDIA). He started his career as a lecturer at Bundelkhand Institute of Engineering and Technology, Jhansi (INDIA). Currently, he is Dean \& Professor in School of ICT at Gautam Budhha University, Noida (INDIA). He was Ex-head of CCN \& ECE Department at National Institute of Technology, Kurukshetra (INDIA). He teaches post-graduate and graduate level courses on Wireless communication and CDMA systems. His research interests include mobility management in cellular / wireless networks, Planning, Designing and optimization of Cellular Networks \& Wireless network security. He has published 47 research papers in International/ National journals and Conferences. Dr. Brahmjit Singh received the Best Research Paper Award from 'The Institution of Engineers (India)' in 2006. 